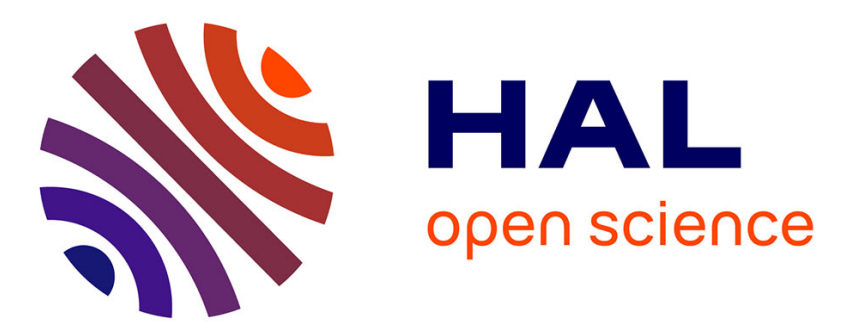

\title{
Un écrit apocryphe attribué à Jérôme: les donatistes, le pape Damase et les origines de la conférence de Carthage de 411 \\ Bruno Pottier
}

\section{- To cite this version:}

Bruno Pottier. Un écrit apocryphe attribué à Jérôme: les donatistes, le pape Damase et les origines de la conférence de Carthage de 411. Xavier Dupuis; Valérie Fauvinet-Ranson; Christophe J. Goddard; Hervé Inglebert. L'automne de l'Afrique romaine. Hommages à Claude Lepelley, Hermann, 2021, 9791037005441. halshs- 03466360

\section{HAL Id: halshs-03466360 \\ https://shs.hal.science/halshs-03466360}

Submitted on 14 Jan 2022

HAL is a multi-disciplinary open access archive for the deposit and dissemination of scientific research documents, whether they are published or not. The documents may come from teaching and research institutions in France or abroad, or from public or private research centers.
L'archive ouverte pluridisciplinaire HAL, est destinée au dépôt et à la diffusion de documents scientifiques de niveau recherche, publiés ou non, émanant des établissements d'enseignement et de recherche français ou étrangers, des laboratoires publics ou privés. 


\title{
Un écrit apocryphe attribué à Jérôme : les Donatistes, le pape Damase et les origines de la conférence de Carthage de 411
}

\author{
Bruno Pottier ${ }^{1}$
}

Deux manuscrits ont conservé le texte d'une lettre apocryphe de Jérôme de Stridon au pape Damase, un document qui est peu connu et a été peu étudié sans doute pour son caractère souvent allusif et confus ${ }^{2}$. Dans cette lettre, Jérôme est supposé critiquer durement Damase, pape entre 366 et 384, pour avoir montré une coupable tolérance envers l'évêque catholique Restitutus de Carthage qui aurait été arien et est même présenté, de manière emphatique, comme un des maîtres d'Arius ${ }^{3}$. Le caractère hérétique de l'évêque de Carthage aurait fait scandale dans tout l'Orient et même dans l'ensemble du monde romain. Il aurait notamment incité lors du concile de Rimini de 359 l'ensemble des évêques africains à renier l'Église de Dieu.

Il s'agit en réalité, comme nous allons le montrer, d'un pamphlet donatiste du début $\mathrm{du} \mathrm{v}^{\mathrm{e}}$ siècle destiné à rappeler les compromissions passées de certains catholiques africains avec l'arianisme. En attribuant à Jérôme des propos hostiles envers Restitutus de Carthage, et plus généralement envers les catholiques africains, l'auteur de ce pamphlet a tenté d'obtenir d'aristocrates proches de ce célèbre auteur chrétien une certaine tolérance envers le donatisme. En effet, certains d'entre eux, notamment ceux qui possédaient des domaines ou qui avaient des proches en Afrique du Nord, se sont investis au début du ve siècle dans la lutte contre ce schisme. D'autre part, l'auteur de cette lettre a pris clairement position en faveur de Jérôme dans le conflit l'opposant à Rufin d'Aquilée, qui était soutenu par d'autres aristocrates chrétiens influents en Afrique du Nord, notamment la famille des Anicii. De plus, certains membres du cercle de Jérôme ont joué un rôle capital dans l'organisation de la conférence de Carthage de 411, qui a réuni l'ensemble des évêques catholiques et donatistes africains et qui a eu pour conséquence une condamnation définitive de ce schisme par le pouvoir impérial. Cette tentative de convaincre certains proches de Jérôme d'observer une certaine

1. Université d'Aix-Marseille, Centre Camille-Jullian.

2. Cette lettre a été éditée et traduite par Maier 1989, p. 198-203. Les deux seules études sur cette lettre sont celles de Morin 1923 et de De Bruyne 1931.

3. Hier., Epistula, 1. 14-22: Quid est hoc quod ab aliqua parte mundi, id est ab Africa, in Oriente resonuit? Verumne est Restitutum Carthaginiensem episcopum magistrum fuisse Arii, non dico discipulum, qui redivivam doctrinam pridem damnatam audeat suscipere, ecclesiam dei Ariminiomnem synodum Africae negare compulerit. 
tolérance envers le donatisme, soit dans les premières années du v viècle soit à l'occasion de cette conférence, est cependant restée infructueuse.

\section{Les donatistes de Rome et le pape Damase}

Les accusations portées dans cette lettre se placent dans le contexte des tentatives de Constance II pour unifier le dogme des églises occidentales et orientales. Restitutus est en effet accusé d'avoir corrompu l'ensemble des évêques africains lors du concile de Rimini qu'il a présidé en juillet 359. Ce concile, voulu par Constance II, a réuni 400 évêques occidentaux. L'auteur de cette lettre pèche cependant par imprécision. En effet, Restitutus ainsi que la majorité des évêques réunis à Rimini ont d'abord rejeté la formule de foi homéenne proposée comme un compromis par certains évêques proches de Constance II qui rejetaient le crédo de Nicée. Convoqué en Thrace par l'empereur avec une délégation du concile, Restitutus a cependant cédé aux pressions impériales et a finalement accepté de signer cette profession de foi ${ }^{4}$. Le concile de Rimini a été donc présenté par Constance II comme une victoire du dogme homéen. Hilaire de Poitiers a particulièrement dénoncé dans son histoire ecclésiastique les responsabilités de Restitutus, un jeune évêque qui comprenait mal les subtilités théologiques discutées lors du concile. Son revirement a été perçu comme une véritable trahison, qui aurait entraîné celle de la majorité des évêques occidentaux ${ }^{5}$. L'auteur de cette lettre apocryphe n'a donc pas totalement déformé la réalité. Or, Restitutus et les autres évêques catholiques africains semblent avoir conservé la foi homéenne de Rimini pendant un long moment après 359, même après la mort de Constance II. En effet, Athanase d'Alexandrie a éprouvé le besoin d'envoyer en 369 une lettre aux évêques catholiques africains pour les inciter à adopter le dogme nicéen ${ }^{6}$. Une lettre de Jérôme récemment découverte évoque la présence à Rome en 382 d'une délégation de l'Église catholique africaine menée par le diacre Aurélius de Carthage. Charles Pietri en a déduit que ce n'est qu'à cette date que Restitutus a abandonné la foi homéenne et s'est finalement rallié au pape Damase qui professait le crédo de Nicée et qui a tenté de supprimer toute forme d'arianisme en Occident ${ }^{7}$. L'auteur de cette lettre apocryphe rappelait cette trahison peu glorieuse des catholiques africains qui avait duré plus de vingt ans. Il importe donc de revenir sur la question de sa datation.

Cette lettre est supposée avoir été envoyée à Damase par Jérôme, alors en Orient, peu avant qu'il devienne son secrétaire en 382. Elle évoque un voyage à Éphèse de Jérôme qui peut avoir effectivement eu lieu lors de son déplacement d'Antioche à Constantinople

4. PCBE, I, Afrique, Restitutus 1, p. 968.

5. Hil., Hist., VIII, 3 (CSEL, 65, 85-86). Un autre évêque africain, Musonius de Byzacène, fut chargé de lire la déclaration finale qui fut approuvée par les participants au concile. Voir Folliet 1966; Merdinger 1997.

6. Athan., Ep. ad Afros, PG, 26, col. 1029-1048.

7. Hier., Ep., 27*, éd. J. Divjak. Cf. Pietri 1983, p. 344. 
entre 379 et $380^{8}$. Elle semble correspondre au contexte de la victoire de la foi nicéenne aux conciles d'Aquilée et de Constantinople de 381. Cependant, Jérôme n'aurait sûrement pas prononcé des attaques aussi dures contre Damase qui l'a pris comme secrétaire, ce qui prouve son caractère apocryphe.

De plus, cette lettre n'a pas été écrite avant les années 401-402 puisque Jérôme est supposé, au mépris de toute cohérence chronologique, se vanter d'avoir ramené son ancien ami Rufin d'Aquilée, participant à une erreur diabolique, dans l'orthodoxie'. Il s'agit d'une allusion au célèbre conflit entre ces deux auteurs ecclésiastiques autour de la pensée d'Origène qui a débuté en 398 et a fortement divisé, comme nous le verrons, l'aristocratie sénatoriale chrétienne. En réalité, Rufin ne s'est jamais soumis aux arguments de Jérôme. L'allusion dans cette lettre apocryphe à sa victoire s'explique sans doute par une référence à un ouvrage de Jérôme, son Apologie contre Rufin, dont le troisième livre rédigé dans les années 401-402 est resté sans réponse. Ce silence pouvait laisser penser à un auteur peu informé que Rufin avait finalement accepté de se soumettre ${ }^{10}$.

L'auteur de cette lettre apocryphe était très probablement donatiste. En effet, comme Donatien de Bruyne l'a remarqué, seul un membre de cette Église schismatique pouvait au début du ve siècle avoir intérêt à rappeler la trahison largement oubliée de Restitutus en $359^{11}$. Or, Restitutus n'a jamais été mis en cause par l'Église catholique africaine et a même été inclus dans le martyrologue de Carthage ${ }^{12}$. Le rappel de cette trahison permettait d'actualiser les accusations portées par les donatistes aux origines du schisme contre deux autres évêques de Carthage, Mensurius et Caecilianus. En effet, ils ont été critiqués comme l'a été plus tard Restitutus pour avoir fait preuve de faiblesse envers le pouvoir impérial lors d'une période de persécution ${ }^{13}$. Restitutus pouvait donc être désigné comme eux en tant que traditor.

Dans cette lettre apocryphe, Jérôme est supposé présenter Damase comme le véritable seigneur des Églises du monde entier. Il lui aurait même donné le titre de monarchita, qui est un hapax, en plus de celui de pape, pour qualifier son pouvoir ${ }^{14}$. En effet, l'épiscopat de Damase a été marqué par une très forte affirmation des droits juridictionnels de la papauté

8. Kelly 1975 , p. $57 ; 68$.

9. Epistula, 1. 45-49: Aut forsitan ignoras talia praesumi vindicanda, sicut Rufini fraus nobis cogentibus patafacta est et verecundia ductus a diaboli laqueo resipuit?

10. D. De Bruyne a situé sa rédaction entre 401 et 405 . Cependant, il ne donne pas d'argument probant imposant que sa date de rédaction ait été antérieure à la condamnation légale du donatisme en 405. Cf. De Bruyne 1931, p. 71-72.

11. De Bruyne 1931, p. 72-73. G. Morin, le premier éditeur du texte, l'a placé par contre au début du viI ${ }^{\text {e }}$ siècle sans argumentation probante (Morin 1923, p. 122-123). Donatien De Bruyne a aussi relevé la présence d'un africanisme dans la langue de cette lettre, l'expression martyrum dicere (voir Maier 1989, p. 198).

12. À la date du III kal. Sept (Depositio Restituti et Augustini). Augustin lui a consacré un sermon, le De depositione episcopi Restituti Carthaginiensis (Possid., Indiculus, 10, 6, 52).

13. PCBE, I, Afrique, Caecilianus 1, p. 166-175; Mensurius 1, p. 748-749.

14. Ce terme n'est pas cité dans le Thesaurus Linguae Latinae. Il peut s'agir d'un africanisme, tout comme l'expression martyrum dicere qui apparaît dans cette lettre. Le Liber Praecum envoyé par des Lucifériens à Théodose en 384 a de même dénoncé la regalis auctoritas de Damase (Collectio Avellana, II, 83, 4-8). 
sur l'ensemble des Églises occidentales. En 378, une constitution de l'empereur Gratien a ainsi accordé au pape une juridiction sur les évêques occidentaux qui lui étaient déférés par des conciles provinciaux. Les préfets du prétoire de Gaule et d'Italie, ainsi que le proconsul d'Afrique, devaient assurer le transfert des évêques inculpés. Cette juridiction concernait donc aussi l'Afrique du Nord ${ }^{15}$. Cette constitution de Gratien répondait à la demande d'un synode romain de 378, qui a évoqué le cas d'un évêque nommé Restitutus, à qui Gratien avait précédemment imposé de venir se justifier à Rome. Une émeute de ses partisans en Afrique du Nord lui avait permis d'échapper à cette convocation ${ }^{16}$. Plusieurs commentateurs ont supposé qu'il s'agissait d'une allusion à l'évêque catholique de Carthage, qui aurait reçu l'injonction de se justifier à Rome de ses prises de position lors du concile de Rimini ${ }^{17}$. Comme Germain Morin l'a remarqué, il semble cependant étonnant dans ce cas que la qualité de primat de Carthage n'ait pas été mentionnée ${ }^{18}$. Cette omission pourrait en fait s'expliquer par l'insoumission de cet évêque, tant envers le pouvoir impérial que l'autorité papale, ainsi que par la suspicion d'hérésie. L'auteur de cette lettre apocryphe peut avoir voulu dénoncer l'inefficacité de Damase, qui n'est pas parvenu en 378 à convoquer Restitutus à Rome, sans avoir eu connaissance du ralliement postérieur de celui-ci en 382, démontré par Charles Pietri ${ }^{19}$. Il pouvait donc critiquer la coupable négligence de Damase envers cet évêque de Carthage.

Cette lettre apocryphe dénonçait donc les prétentions monarchiques de Damase en pointant aussi ses négligences qui semblaient recéler une secrète connivence avec l'arianisme. Il semble ainsi indigne de ses prétentions exagérées. Selon cette lettre, Jérôme aurait de plus accusé Damase de n'avoir rien fait pour lutter contre la très forte diffusion de l'arianisme parmi les évêques de sa propre province, en Italie ${ }^{20}$. Damase et les évêques italiens dans leur ensemble ne pouvaient donc logiquement revendiquer une autorité outre-mer et intervenir dans les conflits religieux africains. L'auteur de cette lettre reprenait en fait des accusations qui ont été portées contre Damase lors de son élection en 366 au trône papal. Son compétiteur Ursinus a dénoncé ses sympathies pour l'arianisme en tant qu'héritier du pape Félix, imposé en 355 ou en 356 par Constance II pour remplacer Libère, fidèle au dogme nicéen ${ }^{21}$. Or, Félix est durement dénoncé en tant qu'arien dans cette lettre apocryphe, même si les informations

15. Pietri 1976, p. 741.

16. Ep. 4-9: Et hoc gloriae vestrae, clementissimi principes : Per Africam quoque Restitutum nomine causam dicere apud episcopos iussit vestra clementia. Debuit acquiescere; sed idem saeva et insolentium manu a causae dicendae necessitate diffugit. Voir Maier 1989, p. 53-54.

17. C. Pietri a notamment défendu cette position contre l'avis d'André Mandouze. La déposition d'un autre évêque compromis lors du concile de Rimini en 359, Urbanus de Forma, a d'ailleurs été confirmée par le rescrit de Gratien de 378. Voir Pietri 1983; Frend 1952, p. 200 ; 206; Grasmück 1964, p. 159, note 891 ; PCBE, I, Afrique, Restitutus 1, p. 968 ; Restitutus 2, p. 969.

18. Morin 1923, p. 123.

19. Voir supra n. 6.

20. Epistula, 1. 37-40.

21. La datation de l'exil de Libère n'est pas assurée. Voir Pietri 1976, p. 249-250; Lizzi 2004, p. 126-144. 
données sur lui sont erronées et confuses ${ }^{22}$. Les donatistes avaient donc connaissance de ces accusations contre Damase, sans doute transmises par des partisans d'Ursinus.

Un autre problème peut expliquer l'hostilité des donatistes envers Damase. Le synode romain de 378 sous la présidence de Damase, qui a convoqué Restitutus à Rome, a aussi demandé à l'empereur Gratien de confirmer son précédent ordre d'expulsion de Claudianus, l'évêque donatiste de Rome, qui n'avait pas été respecté. Claudianus est en effet parvenu à rester évêque donatiste de Rome jusqu'en 384-385, avant d'être expulsé en Afrique ${ }^{23}$. Gratien avait motivé cette mesure par le caractère illicite des rebaptêmes auquel Claudianus avait procédé à Rome et par les calomnies que celui-ci avait proférées contre Damase. Elles sont sans doute relatives au procès de Damase pour adultère de 371-372 ou 375. Cependant, Damase a pu aussi être accusé dès ce moment par Claudianus de sympathies pour l'arianisme. Selon Rita Lizzi, les donatistes de Rome ont donc très probablement appuyé les partisans d'Ursinus dans leurs attaques contre Damase ${ }^{24}$. L'auteur de cette lettre apocryphe aurait repris ces accusations anciennes. Les donatistes ne se limitaient donc pas à un splendide isolement et pouvaient nouer ponctuellement des alliances avec d'autres groupes rigoristes et schismatiques selon les circonstances.

\section{LES DONATISTES ET LE THÈME DE L'UNIVERSALITÉ DE L'ÉGLISE}

L'auteur de cette lettre apocryphe, écrivant après la victoire définitive du dogme nicéen en 381, a tenté de réécrire l'histoire de l'Église au IV siècle. En dénonçant le ralliement de Restitutus à l'hérésie arienne, cet auteur incitait son lecteur à assimiler logiquement les donatistes à des nicéens persécutés par le pouvoir impérial. L'histoire de ce schisme permettait d'accréditer cette présentation, et d'expliquer pourquoi cette Église avait été interdite par le pouvoir impérial, notamment sous le règne de Constance II entre 355 et 361. Or, Jérôme lui-même, dans sa chronique rédigée en 379 ou 380, a évoqué de manière très neutre Donat de Carthage comme étant le fondateur du schisme donatiste, en précisant qu'il s'était diffusé en Afrique et à Rome. Il a mentionné pour l'année 355 son exil dans un passage où il a évoqué la déportation d'évêques nicéens par Constance II, comme Lucifer de Cagliari et ses disciples ${ }^{25}$. Un lecteur peu averti pouvait assimiler Donat à ces héros injustement persécutés

22. Dans une phrase peu claire, l'auteur de cette lettre apocryphe semble supposer que Félix aurait tenté d'usurper le trône de Damase ou seulement qu'il aurait vainement tenté d'être ordonné évêque de Rome à une période non précisée (1. 72-79). Cet auteur semble avoir eu une connaissance limitée de l'histoire de l'Église catholique de Rome. 23. Il est le dernier évêque donatiste de Rome cité dans la seconde édition de l'œuvre d'Optat de Milev ( $A d v$. Parm., II, 4, 5), vers 384-385.

24. Ce procès a été daté de 371-372 par R. Lizzi et de 375 par C. Pietri. Cf. Pietri 1976, p. 420-423; Lizzi 2004 p. 196-203. Voir aussi PCBE, I, Afrique, Claudianus, p. 210; II, Italie, Claudianus, p. 446-447.

25. Il s'est limité à qualifier les donatistes de Rome de sectatores. Hier., Chron., a. 355 : Donatus a quo supra Donatianos in Africa dici memorauimus Carthagine pellitur: quidam sectatores eius etiam montenses uocant eo quod ecclesiam Romae primum in monte habere coeperint. Eusebius Vercellensis episcopus et Lucifer ac Dionysius Caralitanae 
par l'arien Constance II. L'auteur de cette lettre apocryphe pouvait donc espérer au début du $\mathrm{V}^{\mathrm{e}}$ siècle bénéficier d'une certaine tolérance en tant que membre d'une Église certes minoritaire mais persécutée injustement, telle celle des Lucifériens, fondée par Lucifer de Cagliari. En condamnant ce qui pouvait apparaitre au début du v viècle comme des tendances hérétiques passées des catholiques africains, cet auteur pouvait ainsi réintégrer les donatistes à une place prestigieuse au sein de l'histoire de l'Église. La défense dans cette lettre du concile de Nicée, auquel pourtant l'évêque Caecilianus de Carthage a participé, n'était pas contradictoire en effet avec la position des donatistes sur la querelle arienne au début du v viècle ${ }^{26}$. Ils rejetaient en effet à cette époque les fortes ambiguïtés de la théologie trinitaire de Donat de Carthage lui-même en condamnant fermement l'arianisme ${ }^{27}$.

En 408, l'évêque rogatiste Vincentius de Cartenna, membre d'un schisme interne aux donatistes, a suivi la même stratégie pour critiquer Augustin. Il s'est servi d'Hilaire de Poitiers pour évoquer le caractère hérétique des Églises orientales au moment de la crise arienne à l'époque de Constance II, contredisant le principe d'universalité de l'Église catholique ${ }^{28}$. Les ambiguïtés théologiques de Donat ont cependant suscité une autre tentative de réécriture de l'histoire de l'Église, cette fois catholique, dénonçant au contraire les donatistes comme étant des ariens. Cette lettre apocryphe peut avoir eu pour objectif de contredire cette présentation, développée d'abord par Épiphane de Salamine en 376 et par Jérôme lui-même en 393 dans son ouvrage De uiris illustribus. Celle-ci s'est largement diffusée au v viècle tant en Occident qu'en Orient ${ }^{29}$. Cependant, Augustin a lui-même fortement nuancé cette accusation contre les donatistes ${ }^{30}$.

Les critiques très dures contre Rufin d'Aquilée présentes dans cette lettre peuvent surprendre, car elles ne faisaient pas partie du répertoire polémique habituel des donatistes. Rufin est dénoncé durement en tant qu'hérétique, ce qui ne peut faire allusion qu'à son refus de remettre en cause certaines idées d'Origène, comme le lui a demandé Jérôme à partir de 398. Certains donatistes se sont impliqués dans ce débat qui a profondément divisé l'aristocratie romaine chrétienne. Les amis de Jérôme, Pammachius, Marcella et Océanus se sont dans ce contexte fortement opposés aux protecteurs de Rufin, comme Mélanie l'Ancienne, sa petite-fille Mélanie la Jeune et les Turcii Apronianii ${ }^{31}$. Ce débat a aussi gravement divisé

et Mediolanensis ecclesiae episcopi, Pancratius quoque Romanus presbyter et Hilarus diaconus distantibus inter se ab Arianis et Constantio damnantur exiliis. Certains auteurs, comme William Frend et Paul Monceaux, ont déduit à tort de ce passage que Donat était mort en 355. Cf. PCBE, I, Afrique, Donatus 5, p. 301 ; Monceaux 1912, p. 50 ; Frend 1952, p. 181.

26. Epistula, 1. 31-34.

27. Voir notamment Aug., Cresc., II, 4, 4; III, 34, 38; IV, 44, 52; 61, 75.

28. Aug., Ep., 93, 3, 31-32.

29. Epiph., Panarion, 59, 13, 6-8; Hier., Vir. Ill., 93, cités par Maier 1989, p. 48-49; 72.

30. Augustin, beaucoup plus précis, a considéré que Donat n'était pas arien, même s'il ne partageait pas toutes les conceptions catholiques et supposait que le Fils était inférieur au Père. En effet, il défendait le dogme de la consubstantialité (Ep., 185, 1, 1; Haer., 69 2).

31. Epistula, 1. 47-49: sicut Rufini fraus nobis cogentibus patefacta est et verecundia ductus a laqueo resipuit? Cf. Laurence 1996, p. 284-285. 
l'Église impériale et suscité un véritable schisme entre Orient et Occident à partir de la déposition de Jean Chrysostome en 403 de son siège d'évêque de Constantinople. Un des chefs d'accusations présentés contre Jean au concile du Chêne de 403 portait justement sur le soutien qu'il avait apporté à des moines condamnés pour avoir défendu Origène ${ }^{32}$. Plusieurs aristocrates chrétiens amis de Rufin d'Aquilée, comme Mélanie la Jeune et certains membres du groupe familial des Anicii, ont pris parti pour Jean Chrysostome et ont accueilli en Italie certains de ses proches ${ }^{33}$. Un synode romain à la fin de l'année 404 ou au début de l'année 405 a excommunié trois des principaux évêques orientaux, le nouvel évêque de Constantinople, Arsace, Théophile d'Alexandrie et Porphyre d'Antioche ${ }^{34}$. Une loi orientale de novembre 404, sanctionnant ce schisme, a d'ailleurs exclu de l'Église ceux qui refusaient de communier avec ces trois évêques ${ }^{35}$. Cette crise a pris une tournure politique puisque Honorius a exercé de fortes pressions sur son frère Arcadius pour qu'il réhabilite Jean Chrysostome ${ }^{36}$. L'auteur de cette lettre apocryphe a donc pris nettement parti contre les partisans de Rufin d'Aquilée qui se confondaient souvent avec ceux de Jean Chrysostome, comme Mélanie la Jeune et certains des Anicii.

Évoquer cette crise pouvait constituer un argument déterminant pour les donatistes, leur permettant de nier le caractère universel de l'Église catholique. Les allusions à la réprobation de tout l'Orient envers la trahison de Restitutus pouvaient renforcer cette critique. Optat de Milev et surtout Augustin ont dénié toute validité à l'Église donatiste parce qu'elle était surtout présente en Afrique et justifié l'Église catholique parce qu'elle était universelle ${ }^{37}$. Cette universalité reposait principalement pour Augustin sur la communion avec les papes et les Églises d'Orient bénéficiant du prestige d'avoir été fondées par des apôtres ${ }^{38}$. Or, entre 407 et la conférence de Carthage de 411 entre donatistes et catholiques, la communion est restée rompue entre le pape et plusieurs sièges apostoliques orientaux, comme Antioche et Alexandrie ${ }^{39}$. Les évêques catholiques africains étaient d'ailleurs bien conscients de ce problème. Lors du concile de Carthage de juin 407, ils ont en effet demandé au pape de se rapprocher de Théophile d'Alexandrie ${ }^{40}$. Cette lettre apocryphe peut ainsi avoir voulu

32. Voir notamment Van Nuffelen 2013.

33. Pallad., Histoire Lausiaque, 61, 7. Cf. Pietri 2001, p. 301-303.

34. Pietri1976, p. 1321-1325; Dunn 2005.

35. CTh, XVI, $4,6$.

36. Honorius a envoyé trois lettres à Arcadius en 404-405 pour défendre Jean Chrysostome. La question de l'Illyricum constituait cependant à ce moment un autre facteur de tensions entre Orient et Occident. Voir l'Ep. Imp. 38, 8 dans la Collectio Avellana (CSEL, 35) et Pallad., Dial., III, 133. Cf. Demougeot 1951, p. 335-348.

37. Optat., Adv. Parm., II, 1.

38. Voir par exemple Aug., Cresc., II, 46, citant notamment l'Église d'Éphèse.

39. Pallad., Dial., XX, 78. La durée de ce schisme est mal connue. Alexandre, nouvel évêque d'Antioche, a rétabli la communion avec Rome. Son ordination est placée entre 412 et 415 . Atticus de Constantinople a accepté de reconnaître Jean Chrysostome avant la fin de l'année 416, probablement après Alexandre (Theod. Cyr., H. E., V, 34). Cyrille d'Alexandrie a été le dernier évêque oriental à rétablir la communion avec Rome. Cf. Pietri 1976, p. 1327-1338.

40. Cod. Can. Eccl. Afr., 101 (CCL, 149, p. 216-217). 
pointer cette rupture de communion. L'évêque donatiste de Timgad, Gaudentius, a d'ailleurs exigé lors de la conférence de Carthage en 411 que ses opposants prouvent qu'ils étaient en communion avec les autres Églises catholiques ${ }^{41}$. Il est cependant curieux que cet argument déterminant n'ait pas été repris par d'autres auteurs donatistes.

Il est d'ailleurs significatif que Jérôme soit supposé, dans cet écrit apocryphe, avoir constaté lors d'un voyage, supposé implicitement se placer avant son arrivée à Constantinople en 379 ou en 380, le caractère hérétique de l'Église d'Éphèse, fondée par l'apôtre Jean, ainsi que celui de soixante-dix Églises d'Asie Mineure. Ce passage peu clair semble faire allusion à la survie de l'hérésie arienne dans cette région. Une autre interprétation éventuelle peut cependant être proposée. L'auteur de cette lettre pourrait en effet aussi faire allusion à un événement mentionné dans l'acte d'accusation de Jean Chrysostome. Celui-ci a en effet été critiqué pour avoir organisé un concile à Éphèse en 401 ou en 402 réunissant soixante-dix évêques, principalement d'Asie, de Lydie et de Carie, qui aurait déposé à tort six prélats de la première de ces provinces. Il a aussi imposé à Éphèse un de ses proches comme évêque, le diacre Héraclide, qui a été accusé d'être origéniste lors du concile du Chêne ${ }^{42}$. Or, les évêques de la province d'Asie avaient demandé à l'évêque Jean d'intervenir dans leur province notamment parce que celle-ci avait longtemps subi la domination des ariens ${ }^{43}$. L'auteur de cette lettre aurait donc critiqué tant le caractère hérétique de Jean Chrysostome que celui des évêques qu'il avait imposés dans cette province. Il aurait donc dénoncé par cette allusion la diffusion dans celle-ci des idées d'Origène, mises implicitement en rapport avec l'arianisme. Cette diffusion de l'hérésie en Orient ne pouvait que contredire les prétentions de l'Église catholique à l'universalité. Cette allusion peut sembler peu évidente. Cependant, un auteur donatiste peut avoir eu connaissance de ces faits très discutés par les chrétiens contemporains parce que liés à l'acte d'accusation de Jean Chrysostome.

L'auteur de cette lettre apocryphe semble avoir rapproché l'arianisme et l'origénisme, comme Jérôme lui-même l'a fait de manière polémique ${ }^{44}$. Cette allusion montre que certains donatistes n'étaient pas étrangers aux débats contemporains divisant les chrétiens de l'Empire. Cet auteur a expliqué ces critiques en retranscrivant la charge de Jérôme dans une célèbre lettre de 384, largement diffusée et notamment lue par des païens, contre la rhétorique, la philosophie et la dialectique ${ }^{45}$. Cet auteur a développé ce thème en affirmant que ces disciplines, si elles étaient utilisées pour l'étude de la Bible, ne pouvaient conduire qu'à des hérésies, comme celles de Marcion ou d'une figure moins connue, celle du gnostiqueCérinthe de la fin

41. Gesta coll. Carth., III, 102.

42. Pallad., Dial., XIV-XV (SC, 341-342); Socr., H. E., VI, 15; Soz., H. E., VIII, 6, 13. A. Cameron a placé en 402 ce synode habituellement daté de 401. Cf. Cameron et Long 1993, p. 405-408.

43. Pallad., Dial., XIV, 127-139.

44. Duval 1970.

45. Hier., Ep., 22, 30. En 375 ou 376, Jérôme, ermite dans le désert de Chalcis, se serait vu en songe jugé par Dieu qui lui aurait reproché d'être plus un cicéronien qu'un chrétien. 
du premier siècle ${ }^{46}$. Or, la critique de la rhétorique et de la philosophie a été fréquemment employée de manière polémique contre Augustin, ancien rhéteur, par des donatistes comme Pétilien de Cirta ou le grammairien Cresconius ${ }^{47}$.

D'autre part, on peut se demander si l'auteur de cette lettre n'avait pas connaissance des tensions existant entre Jérôme et Augustin, qui aurait été personnellement visé par cette lettre. En effet, Jérôme n'a pas hésité à accuser Augustin, dans une lettre de 404, de tendances hérétiques judéo-chrétiennes parce qu'il avait critiqué certaines de ses traductions bibliques. Il a même significativement critiqué Augustin d'être proche des idées de Cérinthe ${ }^{48}$. Jérôme l'a aussi accusé à tort en 402 d'avoir diffusé à Rome un ouvrage très critique envers lui ${ }^{49}$. Augustin s'est d'ailleurs déclaré épouvanté par le traité de Jérôme contre Rufin ${ }^{50}$. Ces fortes tensions entre les deux intellectuels peuvent expliquer que l'auteur de cette lettre ait choisi d'attribuer à Jérôme de nombreuses critiques contre l'Église catholique africaine. La large diffusion des lettres d'Augustin peut expliquer qu'il ait eu connaissance de ces tensions ${ }^{51}$. La référence à la figure très marginale de Cérinthe dérive peut-être précisément de la lettre de Jérôme de 404, qui aurait été lue par l'auteur de ce document apocryphe. Celui-ci n'avait peut-être pas connaissance de l'hostilité de Jérôme envers les donatistes, attestée au moins à partir de la rédaction du De uiris illustribus en $393^{52}$. Le choix de valoriser la figure de Jérôme, qui est apparemment étrange venant d'un donatiste, peut donc s'expliquer par son hostilité envers Augustin, qui était lui-même proche des positions de Rufin dans la querelle d'Origène. Certains donatistes ont donc pris parti dans ce conflit divisant l'aristocratie chrétienne, en critiquant Rufin et implicitement ses patrons aristocratiques, tels les Anicii.

\section{Augustin et Les AMis de JÉRÔMe}

La volonté d'attribuer une lettre apocryphe hostile à l'Église catholique africaine à Jérôme peut aussi s'expliquer parce que certains de ses proches sont directement intervenus dans la crise donatiste, notamment Pammachius, un de ses amis les plus fidèles ${ }^{53}$. Augustina ainsi envoyé une lettre pour féliciter avec une grande déférence ce célèbre sénateur chrétien pour son intervention. Pammachius a en effet fait lire une exhortation à se convertir à la foi catho-

\footnotetext{
46. Sur Cérinthe, voir Eusèbe de Césarée, H. E., III, 28; Epiph., Pan., XXVIII ; LI, 3; Aug., Haer., 8. L'auteur de cette lettre connaissait peut-être cette hérésie par les écrits de Jérôme lui-même (Vir.ill., 9 daté de 393).

47. Aug., Petil., III, 16, 19; Cresc., I, 2; XIII, 16.

48. Hier., Ep., 112, 13. Jérôme a aussi suspecté en 404 Augustin de manichéisme et d'hérésie (Ep., 105, 5), tout comme l'a fait l'évêque donatiste Pétilien à la même époque. Voir Fry 2010, p. 165-217; Kelly 1975, p. 263-272. 49. Aug., Ep., 67, 2.

50. Aug., Ep., 73, 6; 8.

51. Jérôme $(E p ., 105,1)$ affirme que des lettres d'Augustin qui lui étaient destinées mais qui ne lui étaient pas parvenues étaient largement diffusées à Rome et même disponibles sur une île isolée de l'Adriatique.

52. Hier., Vir.ill., 93.

53. PCBE II, Italie, Pammachius, p. 1576-1581.
} 
lique sur les domaines qu'il possédait au centre de la Numidie consulaire, donc à proximité de $\operatorname{Cirta}^{54}$. Cette curieuse exhortation, qui constituait une sorte de sermon laïc, aurait été très efficace. La datation de cette lettre, attribuée à l'année 401 par les pères mauristes sans argument probant, este difficile ${ }^{55}$. Augustin ne semble pas avoir été lui-même à l'origine de cette action de Pammachius. Celui-ci, proconsul d'Afrique avant 396, peut avoir eu une certaine connaissance du donatisme, notamment par le biais de procès concernant la possession de basiliques qu'il aurait eu à juger. Ceci dénote l'influence religieuse exercée par certains propriétaires terriens, même absentéistes, sur leurs coloni. Augustin a ainsi conseillé à Pammachius de donner à lire sa lettre à certains de ses amis sénateurs chrétiens, pour les inciter à faire de même.

Or, de manière étonnante, Augustin s'est moqué dans cette même lettre de tentatives ridicules de la part de donatistes de rallier à leur cause Pammachius. Celui-ci résidant à Rome à ce moment, cette tentative a pu prendre la forme d'une lettre d'un donatiste africain ou d'une rencontre avec un donatiste de Rome ${ }^{56}$. La lettre apocryphe attribuée à Jérôme, maladroite et peu crédible en soi, pourrait donc constituer un témoignage de tentatives donatistes contemporaines de susciter la sympathie et donc la tolérance de proches de Jérôme. Ces tentatives pouvaient ainsi être fondées sur le rappel des positions hérétiques passées de l'Église catholique africaine et sur la dénonciation de Rufin d'Aquilée et de sa défense d'Origène. Pammachius s'est en effet personnellement opposé avec son ami Océanus à Rufin dès 398 sur cette question ${ }^{57}$. Un membre de la communauté donatiste de Rome, où un évêque schismatique est attesté jusqu'en 411, pourrait donc avoir été l'auteur de cette lettre apocryphe ${ }^{58}$. Or, de multiples lettres apocryphes attribuées à Jérôme circulaient en 401 en Afrique du Nord ${ }^{59}$.

Cependant, il pouvait être dangereux pour des donatistes de prendre position dans un conflit qui a profondément divisé l'aristocratie chrétienne romaine, puisque certains membres des groupes familiaux des Anicii et des Valerii, qui ont joui d'une forte influence à la cour de Stilichon et d'Honorius jusqu'en 408, ont au contraire soutenu Rufin d'Aquilée et Jean Chrysostome dans les années $404-405^{60}$. Si cette lettre apocryphe a été écrite dans les premières années du v siècle et a été largement diffusée à Rome, elle a pu renforcer

54. Aug., Ep., 58, 1 : Verumtamen iam simul, eramus, et coniuncti sub uno capite vivebamus, in cuius caritate nisi radicatus esses, non tibi tam dilecta catholica unitas foret, nec colonos tuos Afros, eo terrarum unde Donatistarum furor exortus est, hoc est in media consulari Numidia constitutos, tali admoneres alloquio, tanto fervore spiritus animares, ut devotione promptissima ad sequendum eligerent, quod te talem ac tantum virum non nisi agnita veritate sequi cogitarent. 55. Selon Brent Shaw, cette lettre doit être datée de la période 405-410, puisqu'Augustin y fait allusion au souci de Pammachius pour le principe d'unité de l'Église $(E p ., 58,1)$. Cependant, cette remarque, très banale chez Augustin, ne fait pas forcément allusion aux lois d'Honorius contre le donatisme de 405. Cette lettre est cependant antérieure à la mort de Pammachius en 410. Cf. Shaw 2011, p. 206, n. 30.

56. Aug., Ep., 58, 3 : Insidias autem quas ipsi haeretici distorto corde moliuntur, quoniam risi eos arbitratos valere aliquid in possessione Christi, animo tuo, nec scribere volui.

57. Laurence 1996, p. 283.

58. PCBE, II, Italie, Felix 20, p. 775.

59. Voir infra note 76.

60. Pietri 2001, p. 301-303; Laurence 2002, p. 139-141. 
l'hostilité de ce groupe d'aristocrates chrétiens envers les donatistes. Ceux-ci, influents à la cour, ont en effet très certainement joué un rôle dans l'interdiction du donatisme par Honorius en $405^{61}$. L'existence d'un schisme en Afrique du Nord fragilisait l'Église catholique occidentale, au moment même où celle-ci entrait dans un autre schisme avec certaines Églises orientales en raison de la déposition de Jean Chrysostome.

\section{Marcellinus, Océanus, le comte Héraclianus et l'organisation DE LA CONFÉRENCE DE CARTHAge EN 4I I}

Une seconde hypothèse est possible. Cette lettre apocryphe peut avoir en effet été rédigée en Afrique du Nord plus tard, vers 411, à l'occasion de la grande conférence organisée par l'empereur Honorius entre les évêques catholiques et donatistes pour résoudre définitivement le schisme. En effet, des proches de Jérôme ont été impliqués dans son organisation. Un point n’a pas été suffisamment souligné. Marcellinus, le tribun et notaire de rang spectabilis choisi par l'empereur Honorius pour diriger cette conférence et juger en dernier ressort, était proche d'Océanus, l'ami de Pammachius, alors réfugié à Carthage comme beaucoup de sénateurs romains fuyant l'invasion d'Alaric. Jérôme a en effet affirmé dans sa lettre 126, dédiée à Marcellinus alors installé en Afrique, que celui-ci était un parens d'Océanus ${ }^{62}$. Ce terme semble devoir être compris plus comme l'indication de liens de parenté réels que d'une direction spirituelle ${ }^{63}$. Jérôme a d'ailleurs affirmé dans cette lettre avoir entretenu

61. Paulin de Nole, correspondant d'Augustin depuis 395et proche de Rufin d'Aquilée, a pu servir d'intermédiaire entre ces aristocrates et l'épiscopat catholique africain. Les délégués du concile de Carthage de 404, Évodius d'Vzalis et Theasius de Memblone, qui ont demandé des mesures de répression contre les donatistes, ont ainsi visité Paulin à Nole avant d'arriver à Ravenne (Aug., Ep., 80, 1). Un autre évêque, Fortunatianus de Sicca, a été envoyé à la cour impériale par le concile de Carthage de juin 408. Il connaissait au moins depuis 405 Paulin qu'il avait visité à Nole (Paul.-Nol., Ep., 3, 3). Possidius de Calama, un ami proche d'Augustin, s'est lui aussi arrêté à Nole pour rencontrer Paulin durant l'été 408 (Aug., Ep., 95, 1). Cf. Hermanowicz 2008, p. 172-173.

62. Hier., Ep., 126, 1 : sanctum parentem vestrum [...] Oceanum. Cette lettre indique qu'Océanus était lui-même présent à Carthage à ce moment.

63. Jérôme a employé le terme parens dans sa correspondance pour qualifier une autorité spirituelle (voir par exemple Ep., 105, 5, pour un évêque, Augustin). Cependant, il est difficile de comprendre pourquoi Marcellinus se serait placé sous la direction spirituelle d'Océanus, qui n'avait pas la réputation d'être un théologien de talent. A. Mandouze a supposé, dans son édition de la $P C B E$, qu'Océanus n'était pas un parent de Marcellinus et n'aurait même pas eu de lien préexistant avec lui, puisque que Marcellinus aurait demandé à Jérôme de le recommander auprès d'Océanus (Ep., 126, 3 : Sanctus frater noster Oceanus, cui vos cupitis commendari). Cependant, la suite de ce passage montre que cette "recommandation" consistait en fait en l'autorisation que Jérôme est supposé donner à Océanus de parler en son nom. Ceci suppose que Marcellinus et Océanus se connaissaient déjà. Jérôme a nié le besoin de cette «recommandation » en ajoutant qu'il considérait qu'Océanus était capable par lui-même de lui exposer sa propre position sur le sujet. L'emploi du terme parens par Jérôme resterait de toute façon à expliquer. P. Laurence a accepté l'existence d'un lien de parenté entre Marcellinus et Océanus. Cf. PLRE, II, Marcellinus 10, p. 711-712; PCBE, I, Afrique, Oceanus, p. 686 note 211; Flavius Marcellinus 2, p. 671-688; 
des contacts épistolaires anciens avec Marcellinus ${ }^{64}$. Il lui a conseillé dans cette même lettre, en réponse à ses questions sur l'origine de l'âme, de s'en remettre à Océanus, suffisamment instruit pour qu'il n'ait pas à lui envoyer des explications sur ce sujet. Il lui a aussi demandé d'emprunter à celui-ci une copie de ses ouvrages contre Rufin, dans lequel il pouvait trouver des éclaircissements. Il a enfin recommandé dans cette même lettre Augustin à Marcellinus pour lui fournir des renseignements complémentaires. Augustin était donc probablement en contact avec Océanus à ce moment ${ }^{65}$.

La datation de cette lettre 126, envoyée à Marcellinus alors en Afrique, est néanmoins difficile à établir. Elle est postérieure au sac de Rome, mentionné comme un événement récent, qui aurait empêché Jérôme de travailler pendant un certain temps. Augustin a affirmé en 418 que Marcellinus l'avait consulté de son propre chef sur le problème de l'origine de l'âme avant de faire appel à Jérôme, qui a répondu par cette lettre $126^{66}$. Or, dans une de ses lettres de 414, Augustin a affirmé ne pas savoir si Marcellinus était déjà installé en Afrique lorsqu'il a envoyé cette demande d'éclaircissements à Jérôme. Cecilaisse pensait qu'Augustin et Marcellinus n'étaient pas encore en contact physique ${ }^{67}$. Il laissait donc entendre qu'il avait déjà entretenu une correspondance avec Marcellinus, soit lorsque celui-ci était encore en Italie en 410, soit peu de temps après qu'il se soit installé en Afrique à la fin de cette année. La lettre 126 de Jérôme peut donc avoir été écrite à ce moment, antérieurement à la conférence organisée à Carthage en juin 411, même si ce n'est pas totalement assuré ${ }^{68}$. Carole Fry a daté cette lettre de l'année 412, en la rapprochant d'une lettre d'Augustin offrant à Marcellinus des éléments de réponse ${ }^{69}$. Cependant, Augustin semble être resté encore très évasif dans cette lettre sur cet épineux problème, qui l'a préoccupé au moins jusqu'en $418^{70}$. Cette lettre d'autre part répond à d'autres préoccupations de Marcellinus et ne se limite pas à la discussion de ce problème. Augustin semble ainsi avoir attendu un certain temps avant de tenter de répondre en 412 aux questionnements de Marcellinus, qui peuvent avoir été exprimés beaucoup plus tôt. Marcellinus a donc eu besoin d'avoir recours à Jérôme. Des contacts épistolaires peuvent donc avoir existé entre Augustin et Marcellinus dès l'année 410 ou le début de l'année 411, peut-être par l'intermédiaire de son parent Océanus ${ }^{71}$.

PLRE, I, Oceanus, p. 636. Ce lien de parenté n'est pas non plus mentionné dans la notice de la PCBE, II, Italie, Oceanus, p. 1547-1549.Voir aussi Laurence 2002, p. 284 note 118.

64. Plusieurs lettres de Jérôme seraient restées sans réponse de la part de Marcellinus (Ep., 126, 1).

65. Hier., Ep., 126, 1.

66. Aug., Ep., 190, 20.

67. Aug., Ep., 166, 8.

68. J. N. Kelly l'a datée de l'année 411 et M. Moreau de la fin de cette année. Cf. Kelly 1975, p. 304; Moreau 1973, p. 81-82.

69. Aug., Ep., 143 (412). Cf. Fry 2010, p. 293.

70. Augustin a en effet envoyé deux lettres à Jérôme sur ce sujet évoquant le questionnement de Marcellinus : Ep., 166 (414); 190 (418).

71. Augustin $(E p ., 151,8)$ a affirmé que la réputation de Marcellinus en tant que chrétien et érudit l'avait précédé avant qu'il ne parvienne en Afrique. Selon M. Moreau, ce passage prouverait qu'Augustin et Marcellinus n'ont pas été en contact avant l'arrivée de ce dernier en Afrique. Il ne s'agit pourtant que d'une remarque élogieuse très 
Pammachius a pu mettre en contact Augustin et Océanus dès 401. Des liens précoces ont pu par ailleurs être établis par l'intermédiaire d'une aristocrate qui semble avoir été une parente d'Océanus, Fabiola, qui possédait vers 422 un domaine dans le castellum de Fussala, à proximité d'Hippone. Augustin a entretenu une correspondance avec elle ${ }^{72}$. De plus, Jérôme a recommandé de manière significative à Marcellinus dans cette même lettre $126 \mathrm{de}$ prendre contact avec Fabiola pour lui emprunter une copie des deux premiers livres de son commentaire sur Ezéchiel ${ }^{73}$. Or, Augustin a envoyé plusieurs lettres à Océanus au moins jusqu'en 416, ce qui montre qu'il a conservé des liens étroits avec ce groupe familial ${ }^{74}$. Jérôme a donc évoqué dans cette lettre un groupe cohérent de réfugiés sénatoriaux, comptant Fabiola et Océanus, qui étaient en contact avec Augustin. Il est alors possible que Marcellinus ait été choisi par le gouvernement impérial pour organiser une conférence contradictoire entre évêques donatistes et catholiques en raison de ses liens de parenté avec Océanus, qui a pu très vite le mettre en relation avec Augustin dès son arrivée en Afrique ${ }^{75}$. La culture religieuse de Marcellinus, ses liens avec Océanus, ainsi que ses contacts avec des autorités reconnues en matière de doctrine chrétienne, le définissaient comme un parfait candidat pour organiser la conférence de Carthage de juin $411^{76}$. Les proches de Jérôme ont donc pu jouer un certain rôle dans l'investissement impérial contre le donatisme à ce moment.

Ces constatations peuvent éclairer le contexte de la lettre apocryphe donatiste attribuée à Jérôme. Celle-ci peut avoir été envoyée, peu avant la conférence de 411, à Océanus ou à Marcellinus, pour contrebalancer l'influence d'Augustin et susciter une certaine tolérance envers les donatistes. En effet, de multiples lettres apocryphes attribuées à Jérôme circulaient à ce moment en Afrique. Jérôme lui-même a accusé en 401 Rufin d'Aquilée d'avoir été l'auteur de certaines d'entre elles, dans lesquelles il aurait critiqué ses propres traductions bibliques $^{77}$. D'autre part, la querelle entre ces deux anciens amis, évoquée dans cette lettre, était bien connue en Afrique du Nord. En effet, Augustin a attesté en 404 de la très large diffusion des écrits de Jérôme contre Rufin dans cette région ${ }^{78}$. Jérôme a aussi mentionné

banale. Selon N. Mac Lynn et B. D. Shaw, Augustin et Marcellinus ne se seraient même rencontrés que lors de la Conférence de Carthage. Cf. Moreau 1973, p. 105-111; Mac Lynn 1999, p. 41 ; Shaw 2015, p. 42 et n. 31. 72. Aug., Ep., 20*, 1;28; 33. Selon Serge Lancel, une autre lettre d'Augustin peut avoir été dédiée à la même personne (Ep., 267). Or, Océanus était parent d'une autre aristocrate romaine nommée elle aussi Fabiola, très proche de Jérôme, et morte entre 398 et 400 . Ces deux aristocrates sénatoriales de même nom étaient probablement apparentées et liées à Océanus. Voir PLRE, II, Fabiola 2, p. 448; PCBE, I, Afrique, Fabiola, p. 380 ; PCBE, II, Italie, Fabiola 1, p. 734-735; Fabiola 2, p. 735; Lancel 1983, p. 278-279.

73. Hier., Ep., 126, $2,2$.

74. Augustin a envoyé une lettre à Océanus en 416 évoquant leur correspondance passée (Ep., 180).

75. L'organisation de cette conférence a été attribuée à Marcellinus par une constitution du 14 octobre 410 (CTh, XVI, 11, 3; Gesta coll. Carth., I, 4; III, 24; 29).

76. Voir le jugement très élogieux d'Orose (Hist., VII, 42, 17).

77. Hier., Ruf., II, 24 (SC, 303). Cet ouvrage a été rédigé en 401-402. Jérôme aurait eu connaissance peu auparavant de l'existence de ce faux par l'intermédiaire d'évêques catholiques africains venus plaider leur cause à la cour impériale. Ceci illustre les liens existant entre Jérôme et ces évêques.

78. Aug., Ep., 73, 6. 
en 402 la circulation en Afrique du traité de Rufin écrit contre lui ${ }^{79}$. Un donatiste africain n'avait donc aucune difficulté à diffuser une polémique contre l'Église catholique locale par le moyen d'une lettre attribuée à Jérôme critiquant notamment Rufin d'Aquilée.

Les circonstances de l'organisation de la conférence de Carthage en juin 411, destinée à résoudre définitivement le problème donatiste à un moment pourtant critique pour l'Occident, sont en fait mal connues. Orose l'a attribuée à Honorius et au patrice Constance, probablement à tort, dans une visée apologétique ${ }^{80}$. Le comte d'Afrique Héraclianus peut par contre avoir joué un rôle décisif. Une loi du 15 janvier 409 a offert la possibilité aux magistrats des cités de faire appel à lui pour prévenir toute sédition contre l'Église catholique en Afrique ${ }^{81}$. Une loi du 25 août 410 lui a été adressée annulant un oraculum impérial qui avait suspendu précédemment les mesures légales contre les donatistes. Cette loi très sévère a condamné à mort toutes les personnes participant à un rassemblement hérétique ${ }^{82}$. Aucun autre exemplaire connu de loi impériale contre les hérétiques, conservé au titre XVI $\mathrm{du}$ Code Théodosien n'a été adressé à un responsable militaire, ce qui souligne son caractère exceptionnel. Ce comte a donc été impliqué dans la répression du donatisme.

Honorius était redevable à Héraclianus du soutien décisif qu'il lui avait apporté face à l'usurpateur Attale en $410^{83}$. L'organisation d'une conférence à Carthage entre évêques donatistes et catholiques peut avoir été une concession de l'empereur à son égard. Elle pouvait avoir pour fonction de récompenser la devotio des Africains catholiques envers l'empereur ${ }^{84}$. Héraclianus a obtenu la fonction de comte d'Afrique en remerciement de sa participation personnelle à l'exécution de Stilichon en août $408^{85}$. Or, ce comte d'Afrique a fait preuve en 410 d'une hostilité prononcée envers certains membres du groupe familial des Anicii réfugiés en Afrique, notamment Anicia Faltonia Proba, la veuve du consul de 371, sa bru Anicia Juliana et sa petite-fille Démétrias, probablement parce que ceux-ci s'étaient compromis avec Stilichon ${ }^{86}$. Anicia Faltonia Proba a notamment entretenu une correspondance avec

79. Hier., Ep., 102, 3.

80. Oros., Hist., VII, 42, 16. En octobre 410, au moment où l'ordre d'organiser la conférence a été donné, Constance avait encore une influence limitée. Sa nomination comme magister militum peut dater d'août 410, en remplacement d'Ellebichus, ou seulement de 411 à l'occasion de la guerre contre Constantin III, qui a constitué pour lui la première occasion de s'illustrer. Orose peut faire en fait allusion à la confirmation en 414 par Honorius, sous l'influence grandissante de Constance, des mesures décidées par Marcellinus contre les donatistes (CTh, XVI, 5, 55). Cf. PLRE, II, Constantius 17, p. 321-325.

81. C. Sirm., 14.

82. CTh, XVI, 5, 51, répétée en CTh, XVI, 5, 56 datée par erreur de 415.

83. Oros., Hist., VII, 42, 10 ; Zos., H. N., VI, 7-10; Soz., H. E., IX, 8. Cf. PLRE, II, Heraclianus 3, p. 539-540; Kotula 1977; Gaggero 1991.

84. Une loi du 25 juin 410 a ordonné une remise d'arriérés d'impôts en Afrique pour récompenser la devotio des Africains envers l'empereur (CTh, XI, 28, 5; 6).

85. Zos., H. N., V, 37, 6.

86. Anicius Petronius Probus a été consul ordinaire en 406 probablement grâce au soutien de Stilichon. Selon Jérôme, Héraclianus a vendu à des marchands syriens comme esclaves des vierges et veuves de naissance noble accompagnant Proba, Anicia Iuliana et Démétrias (Ep., 130, 7). Jérôme a laissé entendre dans cette même lettre une 
Rufin d'Aquilée ${ }^{87}$. Héraclianus avait donc toutes les raisons de bien recevoir au contraire, dès son arrivée en Afrique après le sac de Rome de 410, Océanus, qui s'est fortement opposé à ces aristocrates lors de la querelle autour d'Origène ${ }^{88}$. Celui-ci peut donc avoir usé de son influence à la cour impériale pour obtenir la nomination non seulement de Marcellinus en octobre 410 pour diriger la conférence de Carthage mais aussi de son frère ainé Apringius comme proconsul d'Afrique en 411. Celui-ci était en effet chargé d'appliquer les mesures contre les donatistes qui pouvaient en découler. Ces deux frères ont d'ailleurs ultérieurement été considérés comme compromis avec Héraclianus par le gouvernement impérial. Ils ont été en effet été exécutés le 13 septembre 413 en tant que complices ou satellites du comte rebelle ${ }^{89}$. En outre, Orose a fait l'éloge de Sabinus, le gendre d'Héraclianus, qu'il a loué pour son savoir en utilisant des termes proches de ceux qu'il a employés pour Marcellinus lui-même ${ }^{90}$. Sabinus, qui semble donc avoir été en contact avec Augustin et apprécié par lui, peut avoir conseillé son beau-père pour les questions religieuses. Certains aristocrates chrétiens proches de Jérôme, tel Océanus, ont ainsi pu se voir reconnaître en concertation avec Héraclianus une expertise pour le choix de fonctionnaires pour des postes délicats, supposant tant la conduite de la conférence de Carthage que l'application des mesures contre les donatistes.

La fonction de comte d'Afrique a été attribuée à Héraclianus par son ami Olympius, qui a joué un rôle déterminant dans la chute de Stilichon en août 408 et est alors devenu maître des offices et principal conseiller d'Honorius ${ }^{91}$. Or, Augustin a envoyé à l'automne 408 une lettre assez familière à Olympius pour le louer de sa promotion alors que celle-ci n'était pas encore officielle. Ceci suppose qu'il ait reçu cette information de manière anticipée. La première lettre qu'il lui a adressée ne concernait qu'un problème mineur, le contentieux d'un évêque africain avec le fisc, qui ne supposait nullement que le puissant maître des offices ne s'y implique personnellement. Augustin avait déjà envoyé une lettre à Olympius sur ce sujet avant même qu'il ne devienne maître des offices ${ }^{92}$. Celui-ci, dans sa réponse, s'est déclaré

responsabilité de Proba dans le sac de Rome, aussi mentionnée par Procope (B.V., I, 2, 27). Voir Laurence 2002, p. $142 ; 146-149$.

87. Gennadius, De script. Eccl., 17, PL, 58, col. 1070.

88. Jérôme s'est montré très critique envers Héraclianus, qualifié de tyran dans une lettre à Démétrias $(E p$., 130,7$)$. Cependant, cette lettre a été rédigée en 414, après l'échec de la révolte de ce comte, pour louer cette vierge chrétienne qui était une petite-fille de Proba. Une critique dans cette lettre du comte rebelle était donc parfaitement logique. Jérôme peut avoir eu un avis très différent sur lui en 410 .

89. Aug., Ep., 151, 3-9. Cf. PLRE, II, Apringius 1, p. 123.

90. Oros., Hist., VII, 42, 11; 17.

91. Olympius, introduit à la cour d'Honorius par Stilichon lui-même, était originaire du Pont-Euxin. Il était peut-être venu en Occident avec Théodose en 394. Il a occupé une fonction palatine non précisée avant la chute du maitre de la milice d'origine vandale. Une loi de novembre 408 excluant du Palais tous les fonctionnaires refusant la foi catholique peut être attribuée à ce chrétien intransigeant (CTh, XVI, 5, 42). Cf. Zos., H. N., V, 32, 1 ; PLRE, II, Olympius 2, p. 801-802.

92. Aug., Ep., 96, 1; 97, 1;3. N. Mac Lynn et B. D. Shaw ont mis en avant les difficultés d'Augustin à obtenir le soutien de hauts fonctionnaires. Cependant, leurs jugements sur les rapports entre Augustin et Olympius, jugés 
être au service de l'Église catholique. Augustin en a profité dans une troisième lettre pour demander la confirmation des lois émises à l'époque de Stilichon contre les donatistes avant même la réception d'une ambassade officielle d'évêques africains à Ravenne. Il l'a même chargé de remettre à cette ambassade un mémoire sur les violences donatistes qu'il lui avait fait parvenir avant qu'elle n'arrive en Italie ${ }^{93}$. Augustin considérait donc Olympius comme un auxiliaire de premier plan dans la lutte contre les donatistes, pouvant court-circuiter la procédure habituelle des pétitions épiscopales. Il pourrait donc, par l'intermédiaire de ses bonnes relations avec Olympius, être entré en contact avec Héraclianus dès sa nomination comme comte d'Afrique, ce qui aurait ainsi facilité l'organisation de la conférence de Carthage ${ }^{94}$. D'autre part, déposé par le préfet du prétoire Jovius au début de l'année 409, Olympius a retrouvé sa fonction de maître des offices et joué un rôle de premier plan à la cour d'Honorius l'année suivante ${ }^{95}$. Il a pu lui aussi intervenir pour l'organisation de la conférence de Carthage, en collaboration avec son protégé Héraclianus.

Rien ne prédisposait Augustin à entretenir des relations étroites avec ce puissant maître des offices d'origine orientale. Il n'avait eu auparavant que des contacts réduits ou inexistants avec des hauts fonctionnaires ${ }^{96}$. Cependant, Augustin semble avoir été en contact avec certains membres de cour d'Honorius en 410, notamment avec le nouveau magister memoriae Zénobios. Sa promotion récente s'explique sans doute par sa proximité avec Olympios, lui aussi d'origine orientale. Augustin avait peut-être connu Zénobios lors de son séjour à Milan en $386^{97}$. Cette relation au plus niveau avec Olympius pourrait éventuellement s'expliquer par ses liens avec le réseau des amis de Jérôme, Pammachius et Océanus, qui l'auraient mis en contact avec lui. Ces amis de Jérôme peuvent avoir conseillé Olympius pour certaines questions religieuses. Sigrid Mratschek a proposé d'identifier ce personnage à un homonyme, décrit comme un frater et un vir sanctus, évoqué par Paulin de Nole en 396 dans son hommage funèbre à Paulina, la femme de Pammachius. Ami de ces deux aristocrates chrétiens,

formels et superficiels, doivent être nuancés. É. Rebillard a au contraire insisté sur la précocité et le caractère exceptionnel de cette relation. Cf. Rebillard 1998, p. 137; 145; Mac Lynn 1999, p. 39; Shaw 2015, p. 40-41; 44-45. 93. Aug., Ep., 97, 1; 4 .

94. Aucune documentation ne témoigne de ces liens éventuels. Cependant, Augustin peut avoir expurgé sa correspondance de ses échanges épistolaires avec Héraclianus après la répression de sa révolte de 413.

95. Olympiodore (frg., 8) a affirmé qu'Olympius est redevenu maître des offices avant d'être exécuté par Constance. Il n'a pu récupérer cette fonction qu'après le ralliement de Jovius à Alaric au printemps 410 et peut l'avoir conservée jusqu'en 411. Constance n'a disposé que d'un pouvoir limité à la cour d'Honorius avant 411 (voir supra n. 79). Cf. Lütkenhaus 1998, p. 59-66.

96. La datation de la seule lettre qui pourrait avoir été envoyée avant 408 par Augustin à un haut fonctionnaire, le vicaire d'Afrique Caecilianus, est discutée (Ep., 86).

97. Augustin a répondu en 410 aux questions sur l'histoire de la philosophie posées par Dioscorus, étudiant à Carthage, qui l'a averti de la récente promotion de son frère Zénobios. Dioscorus était aussi en contact à cette époque avec Alypius (Ep., 117-118). Augustin a dédié en 386 son ouvrage De ordine, d'inspiration platonicienne, à un fonctionnaire milanais nommé Zénobios qui fréquentait aussi Alypius. Il pourrait être identifié au magister memoriae de 410 ou éventuellement avoir été un parent de celui-ci. Cf. PLRE, II, Zenobios 1, p. 1196; Dioscorus 2, p. 367; PCBE, I, Afrique, Dioscorus 2, p. 279-280. 
il a prévenu Paulin de ce décès ${ }^{98}$. Cette identification hypothétique permettrait d'expliquer comment un oriental, venu probablement en Occident avec Théodose en 394, a pu nouer des liens avec certains des plus prestigieux sénateurs chrétiens ${ }^{99}$.

Pammachius, même s'il n'a pas assuré de fonctions administratives à cette époque, a bénéficié d'une certaine influence à la cour d'Honorius, notamment en raison de ses compétences doctrinales ${ }^{100}$. Il y a notamment défendu son ami Jérôme, attaqué pour la vision pessimiste de l'Empire qu'il avait donnée en 407 dans son commentaire du livre de Daniel. Jérôme a notamment accusé Rufin d'Aquilée d'avoir joué un rôle prépondérant dans ces attaques. Ces critiques semblent avoir eu lieu avant la chute de Stilichon et peuvent avoir impliqué certains aristocrates chrétiens influents, tels des membres des groupes familiaux des Anicii et des Valerii, liés à Rufin ${ }^{101}$. Elles expliqueraient la virulence de Jérôme à l'égard du maittre de la milice d'origine vandale, dont pourrait découler une certaine sympathie envers l'ascension d'Olympius ${ }^{102}$.

Cette lettre apocryphe montre que tous les donatistes ne se sont pas complus dans un splendide isolement régional. Certains ont compris que leur faible visibilité en dehors de l'Afrique et leur investissement limité dans les débats théologiques contemporains pouvaient prévenir toute tolérance à leur égard. Cette lettre semble avoir constitué une tentative maladroite et tardive, voire désespérée, pour attirer l'attention de certains aristocrates chrétiens influents qui ont été en fait plutôt réceptifs à Augustin. Les donatistes ont donc pris position dans les luttes de factions déchirant l'aristocratie chrétienne. Cependant, la prudence d'Augustin face aux querelles théologiques divisant l'aristocratie chrétienne, comme la crise autour d'Origène, lui a permis de multiplier ses soutiens parmi les aristocrates chrétiens romains ${ }^{103}$. D'autre part, ce type d'intervention constituait une modalité nouvelle des relations de patronage traditionnelles exercées en Afrique par certains grands aristocrates romains qui étaient aussi des grands propriétaires locaux.

98. Paul.-Nol., Ep., 13, 1. À la différence de N. Mac Lynn, S. Mratschek a insisté sur l'étroitesse des liens existant entre Augustin et Olympius. Cf. PCBE, II, Italie, Olympius 2bis, p. 1552; Mratschek 2001, p. 225-226. 99. La réputation de piété d'Olympius explique son ascension politique en 408 (Zos., H. N., V, 32, 1).

100. Ainsi s'expliquerait la loi CTh, XVI, 5, 53 du 6 mars 398 qui a durement condamné à l'exil, assorti de la peine du fouet plombé, Jovinien, un ennemi de Jérôme qui critiquait l'ascétisme. Pammachius s'est fortement impliqué dans ce combat au côté de Jérôme $(E p$., $48 ; 49)$.

101. Jérôme a évoqué ces critiques et le soutien apporté par Pammachius dans une œuvre écrite entre 408 et 410 selon Kelly 1975, p. 299 (Is., XI, praefatio). Voir Demougeot 1952, p. 83-92.

102. Hier., Ep., 123, 16 (409). Il n'a pas hésité à affirmer entre 408 et 410 que la mort de Stilichon a été voulue par Dieu, justifiant ainsi son élimination par Olympius et Héraclianus (Is., XI, praefatio). Pammachius est lui-même mort en 410 lors du siège de Rome par les Goths. Cf. PCBE, II, Italie, Pammachius, p. 1576-1581; PLRE, I, Pammachius, p. 663.

103. Aug., Ep., 82, 2, 23. 